\title{
AbbVie invests in pioneering celiac disease therapy
}

AbbVie and Alvine Pharmaceuticals have teamed up to develop ALV003, which is a novel oral treatment that is currently in Phase II development for patients with celiac disease.

Under the terms of the agreement, AbbVie will make an initial upfront payment of US\$70 million for an exclusive option to acquire either the assets relating to ALV003 or the equity of the company upon successful completion of Phase II development. Alvine will also be entitled to receive a milestone payment upon AbbVie's initiation of Phase III development.

Celiac disease is an acquired autoimmune disorder of the small intestine that develops in genetically susceptible individuals after exposure to dietary gluten, causing intestinal inflammation and chronic gastrointestinal symptoms, with potential complications including malabsorption, osteoporosis, anaemia and malignancy. The pathogenesis is complex. "What we know is that all patients have gluten-specific T cells in the small intestinal lamina propria. These T cells respond to gluten-derived peptides bound to the disease-predisposing molecules, human leukocyte antigen DQ2 (HLA-DQ2) or HLA-DQ8, resulting in the production of pro-inflammatory cytokines," explains Frits Koning, Leiden University Medical Center, the Netherlands. "However, most HLA-DQ2and HLA-DQ8-positive individuals do not develop celiac disease, so there must be additional genetic and environmental factors that trigger disease initiation or affect disease development."

There are currently no approved therapies for the disease, and patients must adhere to a strict lifelong gluten-free diet, which is challenging. "Cereals containing gluten are heavily used in the food industry and products that are naturally gluten-free may become contaminated during food processing," says Koning. "Gluten is also one of the cheapest food proteins and has interesting properties, and so is added to many products, making it difficult to completely avoid."

The development of therapies for the disease is also challenging. "Any therapy

.

would have to be as safe as a strict gluten-free diet. No risk-taking is allowed," says Stefano Guandalini, University of Chicago Celiac Disease Center, Illinois, USA.

ALV003 is composed of two gluten-specific proteases: a modified recombinant version of a Hordeum vulgare (barley) cysteine endoprotease and a modified recombinant version of a Sphingomonas capsulata prolyl endopeptidase. "Gluten is only partially degraded in the stomach and upper intestine, and the remaining fragments are large enough to bind to HLA-DQ," says Koning. "So the general idea is that taking enzymes that can degrade gluten with a gluten-containing meal could help alleviate the disease-inducing properties of gluten by breaking down gluten further so that the fragments can no longer bind to HLA-DQ."

In a Phase lla study involving 41 well-controlled patients with celiac disease, in which individuals were randomized to receive oral ALV003 or placebo daily for 6 weeks at the time of ingestion of $2 \mathrm{~g}$ of gluten, ALV003 was reported to significantly attenuate gluten-induced intestinal mucosal injury. Importantly, no serious adverse events were reported.

,

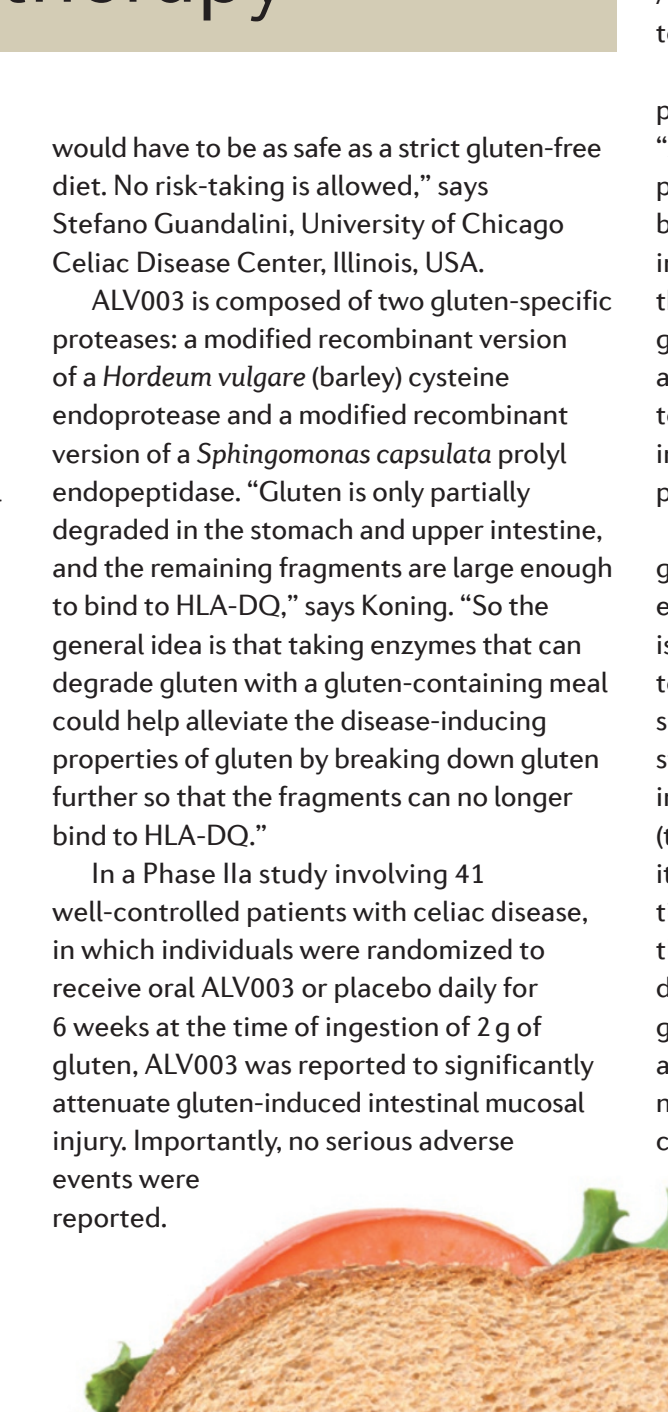

